\title{
Friction and dynamically dissipated energy dependence on temperature in polycrystalline silicon MEMS devices
}

\author{
A. Gkouzou ${ }^{1}$ • J. Kokorian $^{1,2}$ - G. C. A. M. Janssen ${ }^{1}$ - W. M. van Spengen ${ }^{1,3}$
}

Received: 21 August 2017/ Accepted: 4 October 2017/Published online: 20 October 2017

(c) The Author(s) 2017. This article is an open access publication

\begin{abstract}
In this paper, we report on the influence of capillary condensation on the sliding friction of sidewall surfaces in polycrystalline silicon micro-electromechanical systems (MEMS). We developed a polycrystalline silicon MEMS tribometer, which is a microscale test device with two components subject to sliding contact. One of the components can be heated in situ by Joule heating to set the temperature of the contact and thereby control the capillary kinetics at the MEMS sidewalls. We used an optical displacement measurement technique to record the stick-slip motion of the slider with sub-nanometer resolution, and we assessed the friction force with nanonewton resolution. All friction measurements were performed under controlled ambient conditions while sweeping the contact temperature from room temperature to $300{ }^{\circ} \mathrm{C}$, and from $300{ }^{\circ} \mathrm{C}$ to room temperature. We were able to distinguish the two ways in which energy is dissipated during sliding: the 'semi-statically' dissipated energy attributed to asperity deformation and contact yield, and the dynamically dissipated energy
\end{abstract}

The original version of this article was revised due to a retrospective Open Access order.

Electronic supplementary material The online version of this article (doi:10.1007/s00542-017-3575-6) contains supplementary material, which is available to authorized users.

\section{A. Gkouzou}

a.gkouzou@tudelft.nl

1 Department of Precision and Microsystems Engineering, Delft University of Technology, 2628 CD Delft, The Netherlands

2 Philips Medical Systems Nederland NV, Veenpluis 4-6, 5684 PC Best, The Netherlands

3 Falco Systems BV, Remisestraat 1, 2225 TH Katwijk aan Zee, The Netherlands ascribed to the release of the tension in the slider during slip events. We observed an increase in the dynamically dissipated energy at $80{ }^{\circ} \mathrm{C}$ while sweeping down in temperature. This increase is caused by higher adhesion due to capillary condensation between the conformal surfaces. Our study highlights how energy is dissipated during the sliding contact of MEMS sidewalls, and it is helpful in overcoming friction in multi-asperity systems.

\section{Introduction}

Micro-electromechanical systems (MEMS) are miniature devices that combine microscopic mechanical and electrical components. MEMS devices include pressure sensors, accelerometers, actuators, optical and radio-frequency switches (Berman and Krim 2013). Most MEMS to date are fabricated using micromachining processes where either monocrystalline or polycrystalline silicon is used as the structural material. Under ambient conditions, a thin layer of silicon dioxide, called the native oxide layer, is always present on silicon. Moreover, once MEMS structures are exposed to air, water condenses on all surfaces. This is especially important when two surfaces are in close proximity. In that case, capillary menisci are formed that stick the structures together. Their surfaces remain in intimate contact with one another due to attractive capillary forces that contribute significantly to the total adhesion force (Çolak et al. 2012).

In addition to the profound effect of water on the total adhesion force, it is interesting to investigate how capillary condensation affects the friction force between silicon surfaces subject to sliding contact. Atomic force microscopy (AFM) experiments have been carried out to measure friction at the nanoscale, and to unravel the way in 
which water contributes to the stick-slip motion of an atomically sharp silicon dioxide tip against a silicon substrate. The atomically sharp tip can be regarded as one asperity. Thus, the contact between the tip and the substrate is a single-asperity system. At the interface between the tip and the substrate, a capillary meniscus can either enhance friction through increased adhesion or viscoelastic behavior (Kendall 1986; Zhu and Granick 2001; Sirghi 2003), or reduce it through the lubricating properties of water (Binggeli and Mate 1994; Liu et al. 1996). It has been revealed that the effect of water on friction depends on the measurement conditions, such as surface chemistry, relative humidity, and applied normal force (Patton et al. 2001; Asay and Kim 2006; Yu et al. 2012). The velocity and humidity dependence of sliding friction was studied by Riedo et al. (2002). They demonstrated that friction decreases logarithmically with increasing velocity for hydrophilic surfaces, whereas for hydrophobic surfaces, friction increases with increasing velocity. For hydrophilic substrates, higher velocities imply shorter contact times; therefore, the number of capillary menisci forming in the area of contact between two rough surfaces decreases.

Along similar lines, Greiner et al. investigated the same friction dependence on scanning velocity (Greiner et al. 2012). They verified that friction decreases with increasing velocity due to the disruption of the capillary meniscus between the AFM tip and the substrate. Moreover, they explored the friction dependence on the contact temperature between a heated AFM tip and a silicon substrate. It was shown that friction decreases significantly and remains low above $100{ }^{\circ} \mathrm{C}$, due to the evaporation of water from the tip-substrate interface. However, for tip temperatures of $75^{\circ} \mathrm{C}$, friction increases to a maximum, an effect ascribed to faster kinetics that resulted in a more fully developed capillary meniscus between the tip and the substrate. In an earlier work with a friction force microscope (FFM), Jinesh and Frenken concluded that friction increases once capillary-condensed water is present at the contact between a graphite surface and a tungsten tip (Jinesh and Frenken 2006). They also observed that, by increasing the scan range of the tip, the capillary meniscus extends in size and 'writes' a line of immobilized water that lasts several seconds. At the end of this line, the condensate exerts elastic forces on the tip, and friction increases.

AFM/FFM studies have improved our understanding of nanoscale friction, and they have highlighted the influence of capillary-condensed water on the relative motion between two media in single-asperity contact with each other. However, these topics have not been sufficiently addressed in multi-asperity systems with technical relevance, such as MEMS sidewall contacts. When two MEMS structures with rough sidewalls are pressed into each other, the real area of contact is much smaller than the apparent area of contact. Once the sidewalls move against one another, different asperities make contact at different moments in time. Given the brittleness of silicon, these asperities can wear out and introduce debris. Therefore, single-asperity models cannot fully describe friction in rough sidewall surfaces, nor do they predict how many asperities can form capillary menisci. To bridge the knowledge gap between the macroscopic and the atomicscale friction, MEMS test devices have been fabricated to experimentally study friction at this intermediate scale.

The first electrostatic shuffle micromotor was developed by Tas et al. to assess the friction force induced by limited tensile stiffness of clamp feet (Tas et al. 1995). Meanwhile, Senft and Dugger carried out tribological measurements on polycrystalline silicon surface-micromachined test devices, and they were able to determine both the normal and friction forces in such devices (Senft and Dugger 1997). Several years later, Alsem et al. developed a polycrystalline silicon sidewall friction test device, and they performed friction and wear measurements in ambient air (Alsem et al. 2007, 2008). After a long-time operation, they observed that the friction coefficient stabilizes to a steadystate value, while the wear coefficient decreases with increasing number of wear cycles. The aforementioned studies succeeded in measuring friction at the microscale, but they did not examine the effect of temperature on the friction force between two sliding surfaces. In particular, it was still not clear how energy is dissipated during sliding of the MEMS sidewalls, and how it is influenced by capillary-condensed water at the contact of such rough surfaces. Furthermore, regardless of the presence of water, a large number of stick-slip motion cycles can influence the friction behavior of MEMS sidewalls, as the surfaces become more and more conformal during sliding.

In a previous work, we demonstrated that temperatures above $100{ }^{\circ} \mathrm{C}$ reduce the capillary-induced adhesion at the contact between two MEMS sidewalls due to the evaporation of water (Gkouzou et al. 2016). Remarkably, adhesion increases at $75^{\circ} \mathrm{C}$, implying increased density of water at the contact area owing to faster capillary kinetics. Both observations are in agreement with the results of Greiner et al., who showed an increase in the magnitude of the pull-off forces of a heated AFM tip at the same temperature (Greiner et al. 2012). In this work, we explore how capillary condensation affects the sliding friction of two rough microscale surfaces. We developed a new concept of a MEMS device with integrated micro-heaters, which allow the in situ selective increase of the temperature between two sidewall surfaces subject to sliding contact. We performed a large number of stick-slip motion cycles with this device at increasing and decreasing contact temperature, and we assessed how energy is dissipated during sliding. 


\section{Experimental details}

To investigate how high temperatures affect the friction behavior of MEMS sliding sidewalls, we performed friction measurements with a MEMS device, in which the contact area of two sliding components can be heated in situ with micro-heaters. The device was manufactured in a three-layer polycrystalline silicon surface micromachining process by MEMSCAP Inc., known as PolyMUMPs ${ }^{\mathrm{TM}}$. The device consists of two shuttles, the 'ram' and the counter-surface. The motion of the two shuttles is realized by the electrostatic actuation of sets of comb-drive actuators. Figure 1a shows a scanning electron microscope (SEM) micrograph of the MEMS device, and Fig. 1b provides a zoomed-in view of the moving parts. When the counter-surface moves while not in contact with the ram, its displacement is proportional to the actuator voltage squared. We will refer to this voltage-displacement relationship as the 'undisturbed motion'. The counter-surface deviates from this motion when it slides in contact with the ram, because of the friction force acting between the contacting surfaces. While slipping between surface asperities at which it gets temporarily stuck, the countersurface moves in a stick-slip fashion.

We controlled the temperature of the contact area between the counter-surface and the ram by passing an electrical current through the thin parts of the countersurface. To assess the contact temperature, we used microRaman spectroscopy that offers micrometer spatially resolved resolution. The temperature calibration procedure is described in detail in Gkouzou et al. (2016). We measured the position of the counter-surface with subnanometer resolution using an in-house developed optical displacement measurement technique based on curve-fitting (Kokorian et al. 2015).

The same technique was employed to determine the normal force on the contact applied from the ram once it touches the counter-surface. The actuator voltage applied on the ram pushes it forward over $2 \mu \mathrm{m}$, so that it makes contact (snaps in) with the counter-surface at around $100 \mathrm{~V}$. When the voltage is reduced for the ram's backward motion, it temporarily adheres to the counter-surface due to stiction. It is only when the restoring force of the support springs is large enough at around $99 \mathrm{~V}$, that the ram snaps off from the counter-surface. The normal force can be extracted by calculating how much further the ram would have moved in the absence of the counter-surface at the applied actuator voltage. To do this, we fitted a quadratic function to the slope of all forward displacements of the ram to extrapolate the maximum distance per contact temperature. This distance multiplied by the stiffness of the support springs of the ram is the normal force. The stiffness of the ram suspension is $1.0 \mathrm{~N} \mathrm{~m}^{-1}$ (van Spengen and Oosterkamp 2007). Due to thermal expansion while subjected to Joule heating, the counter-surface to ram distance changes slightly. Consequently, for the same actuator voltage applied to the ram, the distance traveled varies with contact temperature (Fig. 2). We calibrated the actuator voltage of the ram as a function of temperature to account for this changing distance, and to ensure that the externally set normal force remains constant at $280 \mathrm{nN}$ throughout the friction measurements.
Fig. 1 a SEM micrograph of the MEMS device used in this study. For each of the two moving components, the 'ram' and the counter-surface, two pairs of beams are used to measure their motion optically. An electrical current flows through the counter-surface by applying voltage to the heating elements connected to it. One set of comb-drive actuators allows the lateral motion of the counter-surface, and another set moves the ram forward until it makes contact with the countersurface. b SEM micrograph zoomed in at the ram and the counter-surface

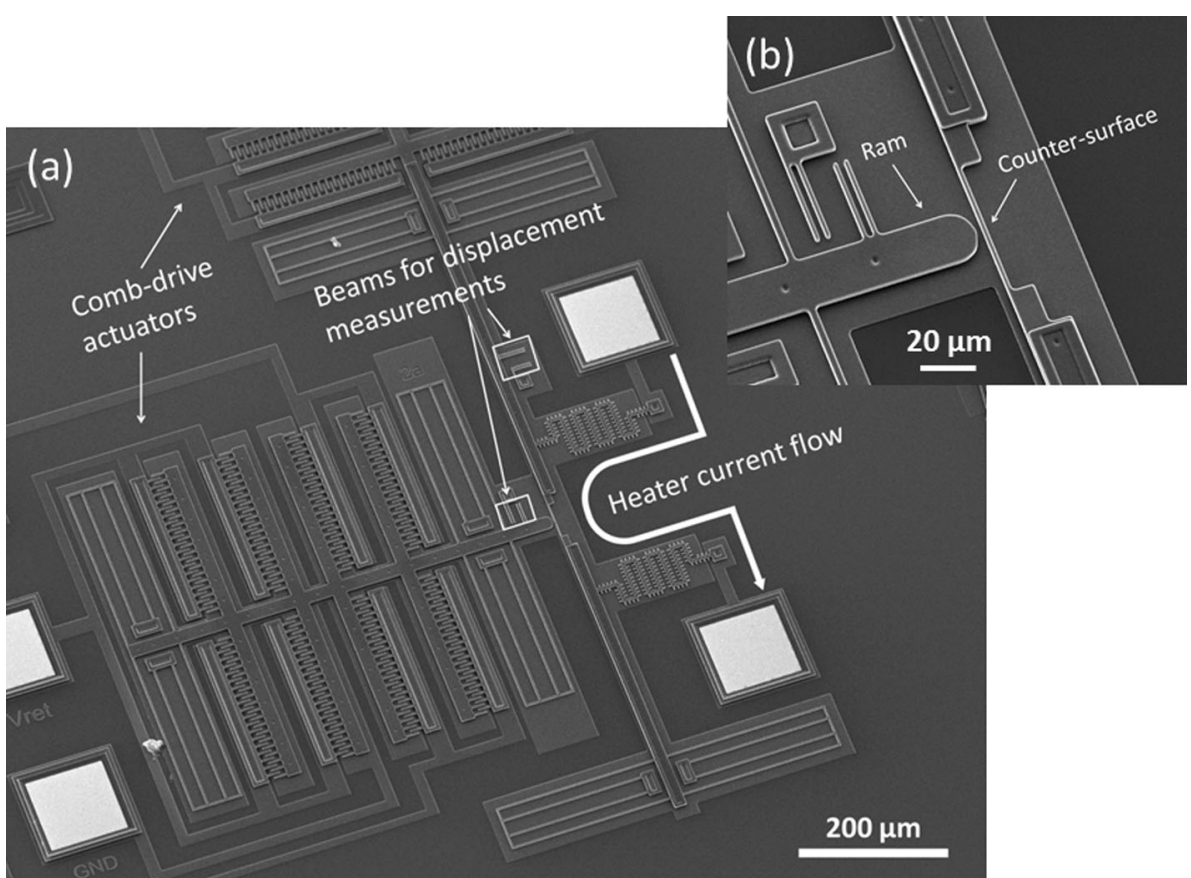


(a) Temperature: $65^{\circ} \mathrm{C}$

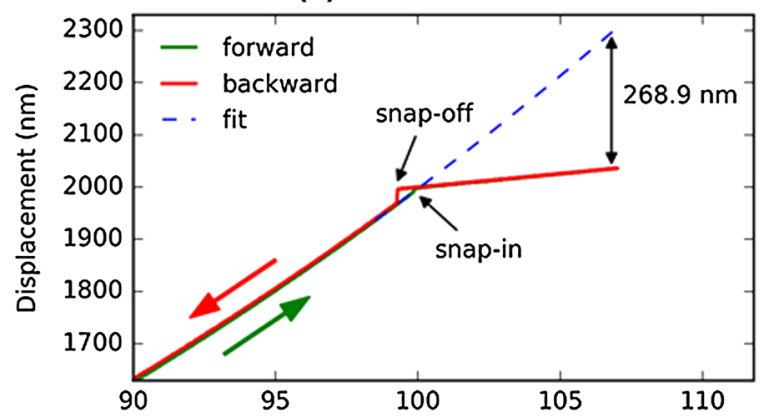

(c) Temperature: $125^{\circ} \mathrm{C}$

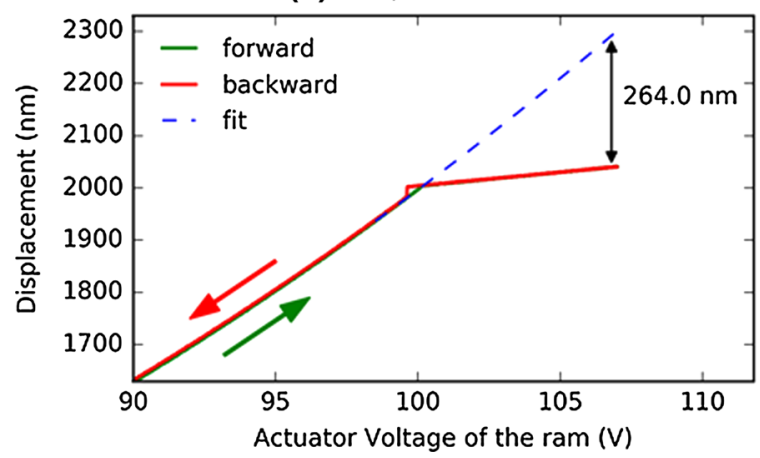

Fig. 2 Measured displacement of the ram as a function of the voltage applied to the comb-drive actuator connected to it at different contact temperatures: a $65^{\circ} \mathrm{C}$, b $80{ }^{\circ} \mathrm{C}$, c $125^{\circ} \mathrm{C}$, and d $175^{\circ} \mathrm{C}$. Due to heating of the contact, the stiffness of the ram changes, resulting in different distance traveled for the same actuator voltage. With increasing actuator voltage, the ram moves forward until it makes contact with the counter-surface (indicated as 'snap in' on the top left figure). Upon decreasing the voltage, the ram remains in contact with the counter-surface until it is released during the backward motion

We used a model of plastic flow contact mechanics to estimate the initial real total area of contact due to multiple asperities of the two polycrystalline silicon surfaces (van Spengen et al. 2002); from the ratio of the normal force to the material effective hardness (5.5 GPa) (Gkouzou et al. 2016), the real area of contact was found to be $50 \mathrm{~nm}^{2}$. The real area of contact between the asperities is expected to increase once the opposite sidewalls slide against one another, causing asperity deformation and wear.

To calculate the friction force, the stiffness of the counter-surface's support springs is required. Due to the complicated geometry of the counter-surface, the spring constant was computed numerically via finite element analysis using COMSOL Multiphysics 4.4. The computed stiffness of the counter-surface was found to be $9.3 \mathrm{~N} \mathrm{~m}^{-1}$. We also computed the lateral stiffness of the ram; with a stiffness of $110 \mathrm{~N} \mathrm{~m}^{-1}$, the ram does not bend laterally when the counter-surface moves against it.

All friction measurements were performed in an environmental chamber under controlled ambient conditions. The room temperature was kept at $26.5 \pm 0.3{ }^{\circ} \mathrm{C}$, and the (b) Temperature: $80^{\circ} \mathrm{C}$

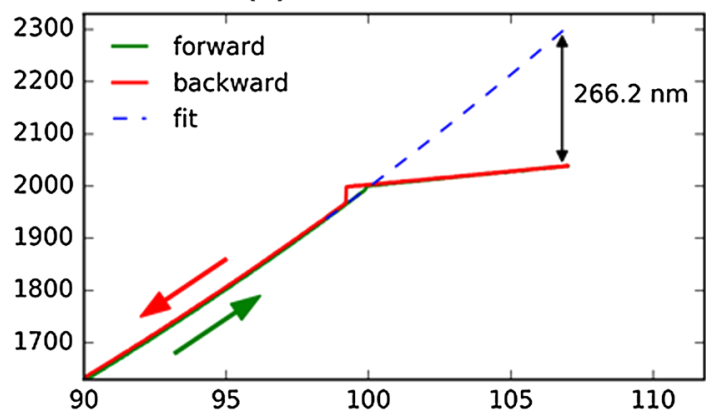

(d) Temperature: $175^{\circ} \mathrm{C}$

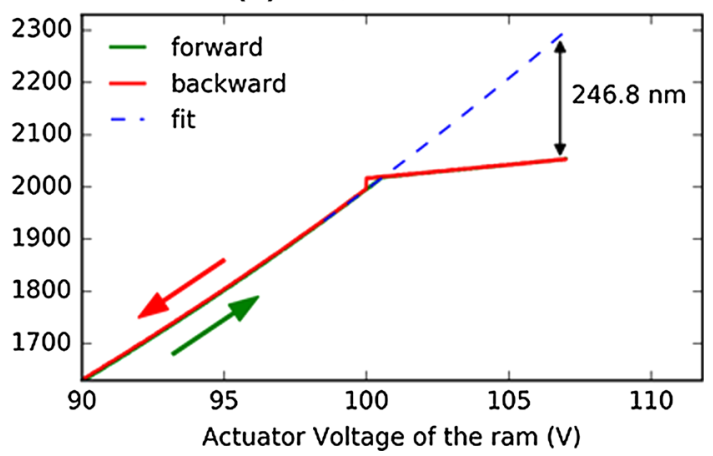

(indicated as 'snap off'). We used a quadratic function to fit the slope of the forward motion and extract the maximum distance at the applied actuator voltage. Note that we are zoomed to a small region of the parabolic curve, which resembles a straight line; yet, the fit is a quadratic function throughout the whole slope. By extrapolating the distance traveled by the ram in the absence of the counter-surface and multiplying it with the stiffness of the ram's support springs, one can determine the normal force

relative humidity was $32.2 \pm 0.4 \%$. Both the temperature and the relative humidity were recorded during all measurements. A detailed data set was obtained in the following way: First, we recorded the undisturbed motion of the counter-surface, with the contact area kept at room temperature. Afterwards, we pushed the ram against the counter-surface with a force of $280 \mathrm{nN}$, and we measured the stick-slip motion of the counter-surface. The measurement of the stick-slip motion was performed 20 times. We increased the temperature of the contact area to $300{ }^{\circ} \mathrm{C}$, in steps of $5{ }^{\circ} \mathrm{C}$. At each temperature step, we measured 20 stick-slip motions, as well as the undisturbed motions of the counter-surface. The contact temperature was then set to room temperature to leave the device in ambient conditions. After three days, we followed the same procedure, but this time with temperatures decreasing from $300{ }^{\circ} \mathrm{C}$ to room temperature. The temperature inside the chamber was kept at $26.4 \pm 0.1{ }^{\circ} \mathrm{C}$, and the relative humidity was $32.8 \pm 0.4 \%$. The sliding velocity of the counter-surface was kept constant at $190 \mu \mathrm{m} \mathrm{s}^{-1}$ during all friction measurements. 
As an example, Fig. 3 shows the displacement of the counter-surface as a function of the actuator voltage at $80{ }^{\circ} \mathrm{C}$ during the undisturbed motion (dashed line), as well as the displacement during a stick-slip motion cycle (solid lines). In this example measurement, the counter-surface starts moving from a position of about $100 \mathrm{~nm}$. This is because none of the previous measurements finished at the position the counter-surface was at the beginning of the experiment $(0 \mathrm{~nm})$ due to the friction force. The difference between the two displacements, multiplied by the stiffness of the counter-surface's support springs, gives us the friction force.

In Fig. 4, we plot the lateral force as a function of the displacement during the undisturbed motion. This is equivalent to the 'support position' that is used for displaying FFM measurements, where it corresponds to the position of the cantilever base. The area enclosed by the forward and the backward curve is the dissipated energy due to the friction force.

In Fig. 5, we introduce a recently proposed alternative representation of friction force loops for MEMS devices, where the lateral force is plotted against the real position of the counter-surface during the stick-slip motion, namely the 'slider position' (Kokorian and van Spengen 2017). Friction in MEMS is best presented when plotted in the manner described above: the measured quantity is the real position of the counter-surface. There is no support position contrary to the FFM case, even though MEMS friction loops have been represented in the past with an artificially created, calculated support position, to mimic AFM-type friction loops (van Spengen and Frenken 2007). The color of the data points corresponds to the magnitude of the

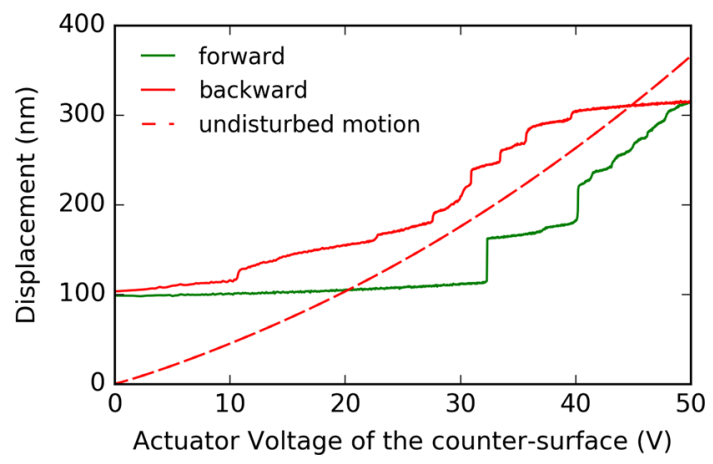

Fig. 3 Measured displacement of the counter-surface as a function of the voltage applied to the comb-drive actuator connected to it, at $80{ }^{\circ} \mathrm{C}$. The dashed line corresponds to the motion of the countersurface while it is not in contact with the ram, the "undisturbed motion'. When the ram pushes against the counter-surface with a normal force of $280 \mathrm{nN}$, the latter moves in a stick-slip fashion in both the forward and the backward direction (solid lines). Note that the displacement of the counter-surface does not start at $0 \mathrm{~nm}$, since this is not the first friction measurement. The counter-surface got stuck elsewhere, and not at the start point, due to the friction force

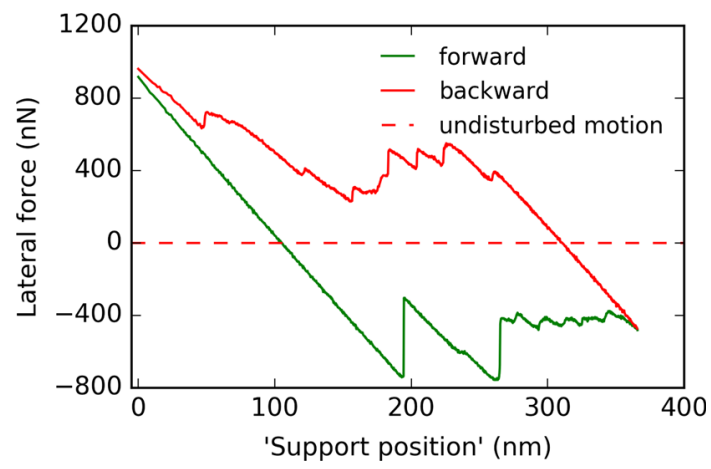

Fig. 4 A MEMS friction loop plotted using the measured positions in Fig. 3, with the friction loop presented in the same way as in typical friction force microscopy (FFM) measurements. The dashed line corresponds to the undisturbed motion, while the solid lines represent the sliding forward and backward motion, with the normal force on the contact being $280 \mathrm{nN}$

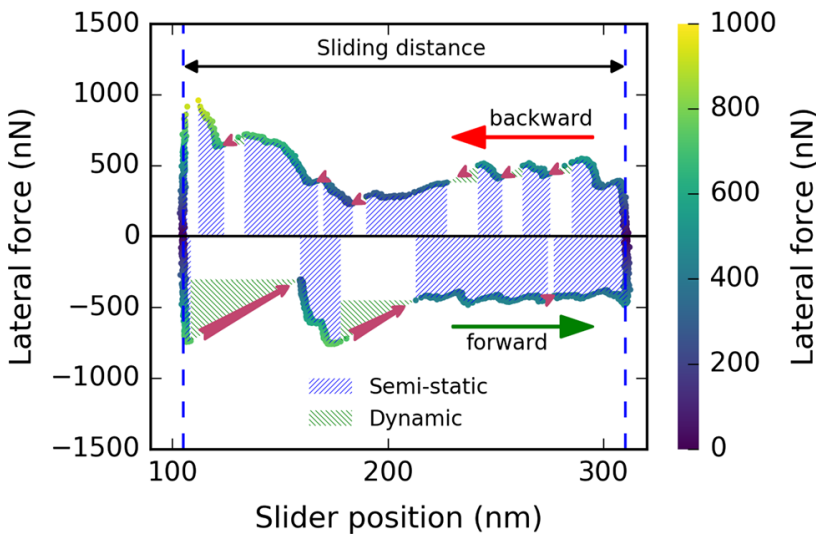

Fig. 5 The lateral force as a function of the real position of the counter-surface during the stick-slip motion. This position is referred to as the 'slider position'. The annotations 'forward' and 'backward' indicate the forward and backward motion of the counter-surface upon increase and decrease of the actuator voltage applied to it, respectively. This is a different representation of the same data presented in the friction loop plotted in Fig. 4. The increase of the lateral force is indicated by the color scale, where it varies from dark to bright. The continuous parts of the curve are the measured positions where the counter-surface is stuck, and the arrows represent the discrete jumps during sliding. The 'sliding distance' is the difference between the minimum and the maximum value of the slider position. The shading indicates the dissipated energy during sliding. The blue area below the continuous parts of the curve corresponds to the 'semistatic energy', and the green area below the arrows corresponds to the 'dynamic energy'

lateral force, and it is meant to give a qualitative impression of the force. We will refer to this plot as a 'friction loop', even though it is not a continuous line, and it is not the familiar AFM-type friction loop, but it has the real position instead of the support position on the horizontal axis. A full discussion of the merits of these new friction loops is given in (Kokorian and van Spengen 2017). The difference between the minimum and the maximum value 
of the slider position within a single friction loop is henceforward referred to as the 'sliding distance'.

The friction measurements with increasing and decreasing temperature generated a total number of 2240 loops. In the supplementary material, we provide images of all friction loops recorded during the experiments. To gain insight of the information provided from these measurements, we analyzed the data in terms of dissipated energy and sliding distance, both as a function of increasing and decreasing contact temperature.

For every loop, we first integrated the area under the continuous parts of the curve, where the counter-surface is in continuous contact with the ram. This area corresponds to the 'semi-statically' dissipated energy due to asperity deformation and contact yield. We will refer to this energy as the 'semi-static energy'. In the semi-static parts, the counter-surface is still stationary (static), but it can exhibit small tangential deflections due to asperity deformation before it slides freely (Luck et al. 2003), a process that is inherently dynamic; hence, we use the term 'semi-static'. In the friction loop, the arrows correspond to discrete jumps (slip events) during the stick-slip motion of the counter-surface. With these arrows, we indicate the motion of the counter-surface while it slips between the deformed asperities. Once the counter-surface jumps, it dissipates energy dynamically due to elastic relaxation of the support springs connected to it; therefore, the area below every arrow from the lateral force value where the counter-surface started the jump to the lateral force value where it got stuck again is a measure of the 'dynamic energy'. This is in fact the difference in stored energy in the support springs between the start and the end of the jump. In Fig. 5, we indicate the areas of the loop that correspond to the semistatic and the dynamic energy. Our technique makes it possible to analyze the obtained friction data with regard to the two ways in which energy is dissipated, due to the fact that we are aware of the exact slider position.

\section{Results}

In Fig. 6, we plot the two types of dissipated energy as a function of temperature of the counter-surface. 20 friction loops were recorded per temperature. The scattered points correspond to the semi-static and dynamic energies of each individual friction loop. The most prominent feature in Fig. 6 is the peak in the dynamic energy at $80{ }^{\circ} \mathrm{C}$ while sweeping down in temperature.

In Fig. 7, we show the sliding distance as a function of temperature of the counter-surface. The error bars that appear in this figure indicate the standard deviation of the 20 measured sliding distances per temperature. Also in Fig. 7, the most distinctive feature is the peak in the sliding distance that appears at $80{ }^{\circ} \mathrm{C}$ when the temperature of the contact was swept from $300{ }^{\circ} \mathrm{C}$ to room temperature.

\section{Discussion}

In Fig. 6, we observe a remarkable increase in dynamic energy at $80^{\circ} \mathrm{C}$ while sweeping down in temperature. The peak appears at around the same temperature in Fig. 7, where we plot the sliding distance as a function of temperature of the counter-surface. We suspect that the dynamics of capillary condensation is responsible for the increase in the dynamically dissipated energy at slightly elevated temperatures. The effect of temperature on capillary condensation is far from trivial. On one hand, higher temperatures favor more water in the gas phase than as condensate on the surface. On the other hand, the "Arrhenius'-type dynamics of capillary meniscus formation dictates that the time it takes to form full 'equilibrium-size' capillary menisci is much shorter at elevated temperatures, increasing adhesion and friction. A full theoretical discussion of these competing effects is beyond the scope of this paper. At the risk of being too speculative, we argue that the formation of a capillary meniscus depends on both the amount of water molecules on the surface, as well as on their mobility. At room temperature, there are many water molecules present on the surface, but they are not very mobile (transport-limited). At a high temperature, the water molecules are very mobile, but only few are present (amount-limited). At the intermediate temperature of $80{ }^{\circ} \mathrm{C}$, sufficient water molecules are present, and they are mobile enough to form fully developed capillary menisci. Therefore, the effect of capillary-induced adhesion is more prominent at this intermediate temperature of $80^{\circ} \mathrm{C}$.

Maximum adhesion occurs at a crossover temperature where capillary condensation is energetically favorable, and where the condensate has sufficient kinetic energy to reach the contact area of the MEMS sidewalls and form capillary menisci. It appears that the optimum temperature is at $80{ }^{\circ} \mathrm{C}$ for our device. As a result, the opposite MEMS sidewalls are pulled closer together, increasing the normal force, and thus the friction force. At the same condition where the dynamically dissipated energy is maximal, the sliding distance shows a maximum as well. This makes perfect sense, since both observations are a consequence of the importance of the slip events during the sliding motion.

There is a huge difference in the dynamically dissipated energy between the experiment with increasing temperature and the experiment with decreasing temperature. We ascribe this difference to the wear of the MEMS sidewalls that causes the surfaces to make more intimate contact after prolonged sliding at elevated temperatures. At the beginning of the experiment, the MEMS sidewall surfaces are 



Fig. 6 The semi-static and the dynamic energy for all individual friction loops as a function of temperature of the counter-surface. For every temperature, there are 20 values that correspond to the

dissipated energy of the 20 individual friction loops. Every blue point corresponds to a semi-static energy, and every green point corresponds to a dynamic energy
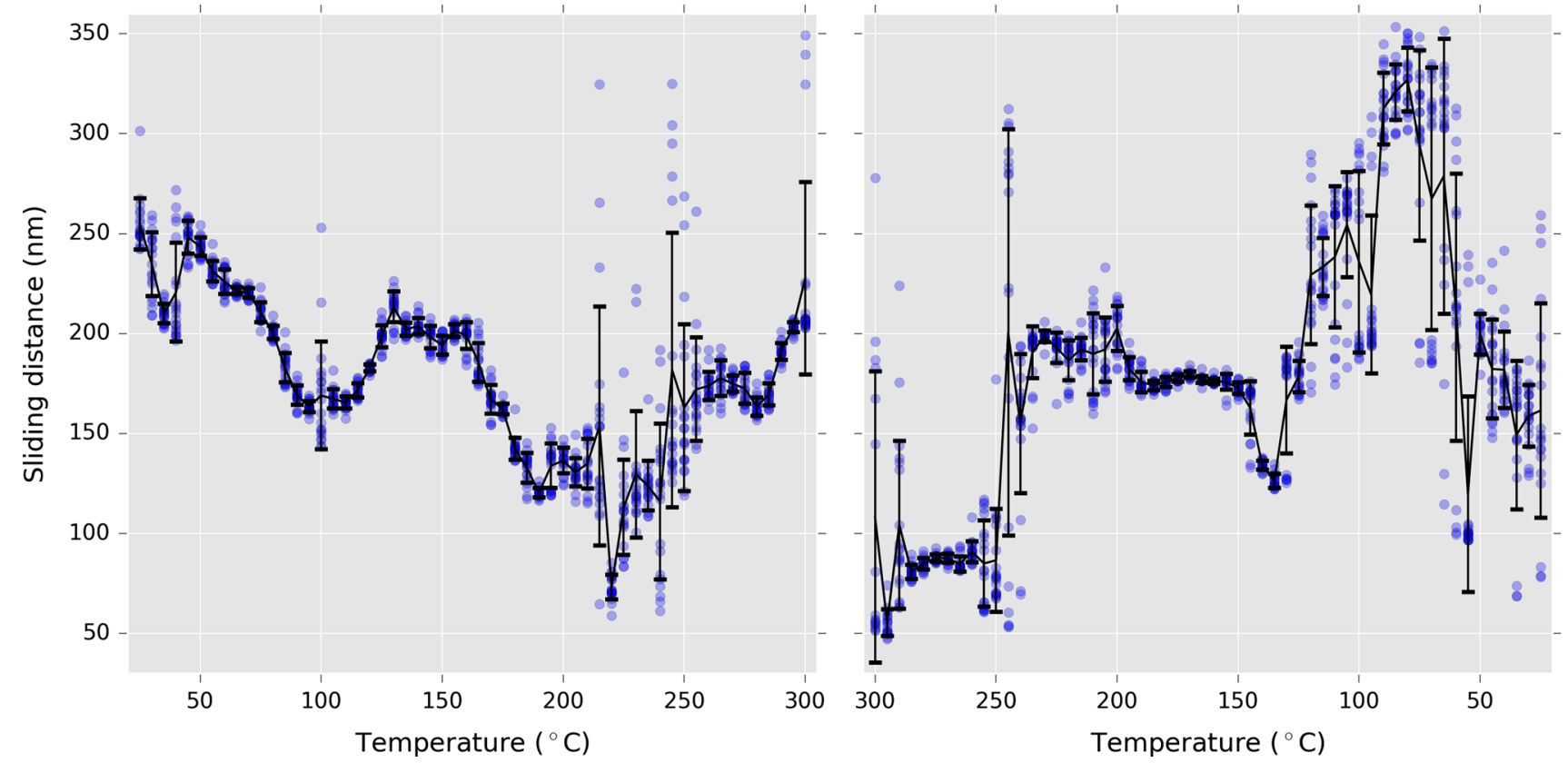

Fig. 7 The sliding distance as a function of temperature of the counter-surface. For every temperature, there are 20 sliding distances. The error bars are not the measured uncertainty; instead, they indicate the variability from cycle to cycle of the stick-slip motion

particularly rough, with the standard deviation of the asperity heights being tens of nanometers (van Spengen et al. 2010). While sweeping down in temperature, a large number of stick-slip motion cycles already elapsed during the experiment with increasing temperature, and the surfaces have become much more conformal due to wear.
More conformal surfaces have a larger area in intimate contact, so more capillary-condensed water is present at their interface. The higher adhesion force that emerges from increased capillary condensation causes the surfaces to become more stuck to one another. The discrete jumps that occur are much larger, so the dynamically dissipated 
energy increases. For rough microscale surfaces, the dynamic energy is low, but it increases once the surfaces have become sufficiently conformal. The fact that the dynamically dissipated energy increases while the semistatically dissipated energy stays roughly the same illustrates that the latter does not severely depend on the amount of adhesion between the surfaces.

In this study, the encountered temperature of $80{ }^{\circ} \mathrm{C}$ is close to the one found in a previous paper, where we investigated adhesion as a function of temperature. In our earlier work, we demonstrated that the adhesion force increases at $75^{\circ} \mathrm{C}$, an effect attributed to a similar faster capillary kinetics (Gkouzou et al. 2016). The results obtained for a MEMS device with sliding surfaces are in full agreement with this earlier observation; therefore, the process of increased capillary kinetics can influence the friction behavior of MEMS sidewalls when they conform well to one another.

\section{Conclusions}

In this work, we have revealed the friction dependence on contact temperature in a MEMS device with sliding components. In line with our previous reported work on capillary-induced adhesion, friction is not constant with temperature; on the contrary, it increases after a large number of stick-slip motion cycles at elevated temperatures that are still within the range where these devices are normally expected to operate, and where capillary condensation is more likely to occur. At a relative humidity of $32 \%$ and at $80{ }^{\circ} \mathrm{C}$, for conformal MEMS sidewall surfaces, a maximum in the dynamically dissipated energy is observed together with a maximum in the sliding distance. The co-occurrence of these two maxima is explained from an increase in the water density at the contact area. Remarkably, while the dynamically dissipated energy increases, the semi-statically dissipated energy remains more or less constant. This means that the device will not only show an increase in the friction force, but it will also exhibit a more erratic stick-slip motion. This work shows that, for MEMS devices with sliding surfaces that are operated in an environment in which water may be present-such as in a non-hermetic package or in open air, as is the case here-the friction force should not be taken for granted after it has been measured once. Our study highlights important details about energy dissipation in MEMS devices, and it can help to bridge the gap between what is known on friction at the macroscale and the valuable information gained from AFM/FMM studies on atomicscale friction.
Acknowledgements This work is part of the research program 'Fundamental aspects of friction' under ref no. 129 of the NWO Institute Organisation (NWO-I), which is part of the Netherlands Organisation for Scientific Research (NWO).

Open Access This article is distributed under the terms of the Creative Commons Attribution 4.0 International License (http://crea tivecommons.org/licenses/by/4.0/), which permits use, duplication, adaptation, distribution and reproduction in any medium or format, as long as you give appropriate credit to the original author(s) and the source, provide a link to the Creative Commons license and indicate if changes were made.

\section{References}

Alsem DH, Stach EA, Dugger MT, Enachescu M, Ritchie RO (2007) An electron microscopy study of wear in polysilicon microelectromechanical systems in ambient air. Thin Solid Films 515:3259-3266

Alsem DH, Dugger MT, Stach EA, Ritchie RO (2008) Micron-scale friction and sliding wear of polycrystalline silicon thin structural films in ambient air. J Microelectromech Syst 17:1144-1154

Asay DB, Kim SH (2006) Effects of adsorbed water layer structure on adhesion force of silicon oxide nanoasperity contact in humid ambient. J Chem Phys 124:174712

Berman D, Krim J (2013) Surface science, MEMS and NEMS: progress and opportunities for surface science research performed on, or by, microdevices. Prog Surf Sci 88:171-211

Binggeli M, Mate CM (1994) Influence of capillary condensation of water on nanotribology studied by force microscopy. Appl Phys Lett 65:415-417

Çolak A, Wormeester H, Zandvliet HJW, Poelsema B (2012) Surface adhesion and its dependence on surface roughness and humidity measured with a flat tip. Appl Surf Sci 258:6938-6942

Gkouzou A, Kokorian J, Janssen GCAM, van Spengen WM (2016) Controlling adhesion between multi-asperity contacting surfaces in MEMS devices by local heating. J Micromech Microeng 26:095020

Gkouzou A, Kokorian J, Janssen GCAM, van Spengen WM (2017) Temperature calibration, normal force measurements, undisturbed motion measurements, and stick-slip motion cycles in MEMS devices with sliding sidewall surfaces. figshare. doi:10. 6084/m9.figshare.4954523.v1

Greiner C, Felts JR, Dai Z, King WP, Carpick RW (2012) Controlling nanoscale friction through the competition between capillary adsorption and thermally activated sliding. ACS Nano 6:4305-4313

Jinesh KB, Frenken JWM (2006) Capillary condensation in atomic scale friction: how water acts like a glue. Phys Rev Lett 96:166103

Kendall K (1986) Inadequacy of Coulomb's friction law for particle assemblies. Nature 319:203-205

Kokorian J, van Spengen WM (2017) Improved analysis and visualization of friction loop data: unraveling the energy dissipation of meso-scale stick-slip motion. Meas Sci Technol. doi:10.1088/1361-6501/aa870a

Kokorian J, Buja F, van Spengen WM (2015) In-plane displacement detection with picometer accuracy on a conventional microscope. J Microelectromech Syst 24:618-625

Liu Y, Evans DF, Song Q, Grainger DW (1996) Structure and frictional properties of self-assembled surfactant monolayers. Langmuir 12:1235-1244

Luck DL, de Boer MP, Ashurst WR, Baker MS (2003) Evidence for pre-sliding tangential deflections in MEMS friction. In: 
Proceedings of 12th IEEE international conference on solid-state sensors, actuators and microsystems (TRANSDUCERS) 1, pp 404-407

Patton ST, Cowan WD, Eapen KC, Zabinski JS (2001) Effect of surface chemistry on the tribological performance of a MEMS electrostatic lateral output motor. Tribol Lett 9:199-209

Riedo E, Lévy F, Brune H (2002) Kinetics of capillary condensation in nanoscopic sliding friction. Phys Rev Lett 88:185505

Senft DC, Dugger MT (1997) Friction and wear in surfacemicromachined tribological test devices. In: Proceedings of SPIE 3224, micromachined devices and components III, Austin, TX, Sept. 5, pp 31-38

Sirghi L (2003) Effect of capillary-condensed water on the dynamic friction force at nanoasperity contacts. Appl Phys Lett $82: 3755-3757$

Tas NR, Legtenberg R, Berenschot JW, Elwenspoek MC, Fluitman JHJ (1995) The electrostatic shuffle motor. In: Proceedings of MME'95, Copenhagen, Denmark, Sept. 3-5, pp 128-131

van Spengen WM, Frenken JWM (2007) The Leiden MEMS tribometer: Real time dynamic friction loop measurements with an on-chip tribometer. Tribol Lett 28:149-156 van Spengen WM, Oosterkamp TH (2007) A sensitive electronic capacitance measurement system to measure the comb drive motion of surface micromachined MEMS devices. J Micromech Microeng 17:828-834

van Spengen WM, Puers R, De Wolf I (2002) A physical model to predict stiction in MEMS. J Micromech Microeng 12:702-713

van Spengen WM, Turq V, Frenken JWM (2010) The description of friction of silicon MEMS with surface roughness: virtues and limitations of a stochastic Prandtl-Tomlinson model and the simulation of vibration-induced friction reduction. Beilstein $\mathbf{J}$ Nanotechnol 1:163-171

Yu J, Kim SH, Yu B, Qian L, Zhou Z (2012) Role of tribochemistry in nanowear of single-crystalline silicon. ACS Appl Mater Interfaces 4:1585-1593

Zhu Y, Granick S (2001) Rate-dependent slip of Newtonian liquid at smooth surfaces. Phys Rev Lett 87:096105 